\title{
Identification of novel $R M R P$ mutations and specific founder haplotypes in Japanese patients with cartilage-hair hypoplasia
}

\author{
Yuichiro Hirose $\cdot$ Eiji Nakashima $\cdot$ Hirofumi Ohashi $\cdot$ Hiroshi Mochizuki · \\ Yuki Bando - Tsutomu Ogata Masanori Adachi $\cdot$ Emi Toba \\ Gen Nishimura $\cdot$ Shiro Ikegawa
}

Received: 18 April 2006/ Accepted: 10 May 2006/Published online: 11 July 2006

(C) The Japan Society of Human Genetics and Springer-Verlag 2006

\begin{abstract}
Cartilage-hair hypoplasia $(\mathrm{CHH})$, or metaphyseal dysplasia, McKusick type, is an autosomal recessive disease with diverse clinical manifestations.
\end{abstract}

Yuichiro Hirose and Eiji Nakashima contributed equally to this work.

Y. Hirose $\cdot$ E. Nakashima $\cdot$ S. Ikegawa $(\square)$

Laboratory for Bone and Joint Diseases,

SNP Research Center,

RIKEN, 4-6-1 Shirokanedai, Minato-ku,

Tokyo 108-8639, Japan

e-mail: sikegawa@ims.u-tokyo.ac.jp

H. Ohashi

Division of Medical Genetics,

Saitama Children's Medical Center, Iwatsuki, Japan

H. Mochizuki

Division of Endocrinology and Metabolism,

Saitama Children's Medical Center, Iwatsuki, Japan

Y. Bando

Department of Pediatrics, Kitasato University

School of Medicine, Sagamihara, Japan

T. Ogata

Department of Endocrinology and Metabolism,

National Research Institute for Child Health and

Development, Tokyo, Japan

M. Adachi

Department of Endocrinology and Metabolism,

Kanagawa Children's Medical Center, Yokohama, Japan

E. Toba

Department of Pediatrics, Tokyo Metropolitan Hachioji

Children's Hospital, Hachioji, Japan

G. Nishimura

Department of Radiology, Tokyo Metropolitan Kiyose

Children's Hospital, Kiyose, Japan
$\mathrm{CHH}$ is caused by mutations in $R M R P$ (ribonuclease mitochondrial RNA processing), the gene encoding the RNA component of the ribonucleoprotein complex RNase MRP. A common founder mutation, 70A $>\mathrm{G}$ has been reported in the Finnish and Amish populations. We screened 11 Japanese patients with $\mathrm{CHH}$ for $R M R P$ mutations and identified mutations in five probands, including three novel mutations (16-bp dup at $+1,168 \mathrm{G}>\mathrm{A}$, and $217 \mathrm{C}>\mathrm{T}$ ). All patients were compound heterozygotes for an insertion or duplication in the promoter or 5'-transcribed regions and a point mutation in the transcribed region. Two recurrent mutations were unique to the Japanese population: a 17-bp duplication at +3 and $218 \mathrm{~A}>\mathrm{G}$. Haplotype analysis revealed that the two mutations common in Japanese individuals were contained within distinct haplotypes. Through this analysis, we have identified a unique mutation spectrum and founder mutations in the Japanese population.

Keywords $R M R P \cdot$ Mutation ' Founder haplotype · Japanese $\cdot$ Cartilage-hair hypoplasia

\section{Introduction}

Cartilage-hair hypoplasia (CHH; MIM 250250), or metaphyseal dysplasia, McKusick type, is an autosomal recessive disease that has diverse clinical manifestations. The most prominent features are short stature and hypoplastic hair. Other common features include metaphyseal dysplasia of the long and short tubular bones, defective immunity, predisposition to malignant tumors including lymphoma, ligamentous laxity, hypoplastic anemia, and Hirschsprung disease. 
Variations in clinical severity are remarkable both between and within families (Makitie and Kaitila 1993). In addition, CHH variants with only skeletal manifestations (metaphyseal dysplasia without hypotrichosis: MIM 250460) have been reported (Verloes et al. 1990; Castriota-Scanderbeg et al. 2001; Bonafe et al. 2002; Nakashima et al. 2003).

$\mathrm{CHH}$ is caused by mutations in the RMRP gene (Ridanpaa et al. 2001), which encodes a 267-nucleotide RNA component of RNase MRP (RMRP: ribonuclease mitochondrial RNA processing) ( $\mathrm{Ri}-$ danpaa et al. 2001; Bonafe et al. 2002). To date, 73 RMRP mutations have been reported (Ridanpaa et al. 2001, 2002; Bonafe et al. 2002, 2005; Nakashima et al. 2003; Kuijpers et al. 2003; Harada et al. 2005; Hermanns et al. 2005; Thiel et al. 2005). The $70 \mathrm{~A}>\mathrm{G}$ mutation is the most prevalent; it comprises $92 \%$ of mutations seen in the Finnish population and is commonly seen in other populations (Ridanpaa et al. 2002). RMRP mutations also cause a variant form of $\mathrm{CHH}$ (Bonafe et al. 2002), anauxetic dysplasia (MIM 607095) (Thiel et al. 2005), and Omenn syndrome (Roifman al. 2006). Mutations in RMRP can be classified into three categories according to the position of the mutation, described as follows: (1) promoter mutations involve duplication or insertion of several nucleotides between the TATA box and the transcription initiation site; (2) transcript mutations are one- or two-nucleotide changes within the transcribed region; (3) $5^{\prime}$ end mutations are insertions or duplications in the $5^{\prime}$ end of the transcript (Ridanpaa et al. 2002). The molecular pathogenesis of these mutations and genotype-phenotype associations are yet to be clarified.

To explore further the range of $R M R P$ mutations in $\mathrm{CHH}$, we examined the RMRP gene in Japanese $\mathrm{CHH}$ patients. Our study reveals several novel and common mutations, and characterizes a unique mutation spectrum and founder mutations in the Japanese population.

\section{Subjects and methods}

Patients and mutation analysis

A total of $11 \mathrm{CHH}$ patients were included in this study. The inclusion criteria were generalized metaphyseal dysplasias of long and short tubular bones with or without extra-skeletal disorders known to be associated with $\mathrm{CHH}$, including hair hypoplasia and immunologic disorders. $R M R P$ mutations had previously been identified in two patients $(\mathrm{CHH} 1,2)$ (Nakashima et al. 2003).

Peripheral blood, hair, or fingernails were obtained with informed consent from the patients and their parents. Genomic DNA was extracted using standard procedures. Polymerase chain reaction (PCR) direct sequencing analysis for $R M R P$ was performed as described (Nakashima et al. 2003).

\section{Haplotype analysis}

Seven patients (CHH1, 2, 4-8) who had RMRP mutations common in Japanese $\mathrm{CHH}$ were subjected to haplotype analysis. We constructed linkage disequilibrium (LD) blocks containing the RMRP gene using genotype data from 44 Japanese individuals in the HapMap phase I database (International HapMap Consortium 2005). The haplotype structure with its tag SNPs was determined using Haploview (Barrett et al. 2005). We genotyped five tag SNPs using the Taqman assay with a PRISM 7900 sequence detector (Applied Biosystems). Haplotypes for chromosomes harboring the two common Japanese mutations were determined by genotyping tag SNPs for seven patients and their parents.

\section{Results}

Identification of $R M R P$ mutations

We screened nine patients for RMRP mutations and identified mutations in six (Table 1). Of these patients, four had hair hypoplasia or immunological disorders, and two showed skeletal changes only ( $\mathrm{CHH}$ variant). Three mutations were novel, and three were recurrent (Nakashima et al. 2003; Bonafe et al. 2005). Other than $182 \mathrm{G}>\mathrm{T}$, these mutations have not been seen in populations other than the Japanese. We suppose that the three novel mutations are not polymorphisms because they were not detected in 65 Japanese controls. Four mutations were point mutations in the transcribed region, and two were duplications in the promoter region. All patients were compound heterozygotes.

\section{Genotype-phenotype association}

Eight Japanese patients with $R M R P$ mutations were evaluated. None of these patients possessed the full spectrum of skeletal, hair, and immunological disorders characteristic of classical CHH. Three had skeletal changes only ( $\mathrm{CHH}$ variant), and hair hypoplasia was 
Table $1 R M R P$ mutations and clinical phenotypes

\begin{tabular}{|c|c|c|c|c|c|c|c|c|c|c|}
\hline \multirow[t]{2}{*}{ Patient } & \multirow[t]{2}{*}{ Age } & \multirow[t]{2}{*}{ Sex } & \multicolumn{2}{|l|}{ Mutation } & \multicolumn{6}{|c|}{ Clinical phenotype } \\
\hline & & & Paternal allele & Maternal allele & $\mathrm{BH}$ & BW & $\mathrm{PH}$ & $\mathrm{SC}$ & $\mathrm{HH}$ & ID \\
\hline $\mathrm{CHH} 1^{\mathrm{a}}$ & 17 & $\mathrm{~F}$ & $182 \mathrm{G}>\mathrm{A}^{\mathrm{b}}$ & 17-bp dup at +3 & 46.5 & 2,900 & -10.0 & + & - & + \\
\hline $\mathrm{CHH} 2^{\mathrm{a}}$ & 7 & $\mathrm{~F}$ & $17-b p$ ins at -20 & $218 \mathrm{~A}>\mathrm{G}$ & 48.0 & 3,200 & -3.2 & + & - & - \\
\hline СHH3 & 14 & M & 16-bp dup at +1 & $168 \mathrm{G}>\mathrm{A}$ & 42.5 & 2,160 & -8.0 & + & - & + \\
\hline $\mathrm{CHH} 4$ & 12 & M & $217 \mathrm{C}>\mathrm{T}$ & $218 \mathrm{~A}>\mathrm{G}$ & 49.0 & 3,000 & -2.0 & + & - & - \\
\hline CHH5 & 8 & $\mathrm{~F}$ & 17-bp dup at +3 & $218 \mathrm{~A}>\mathrm{G}$ & 48.2 & 3,138 & -2.9 & + & - & - \\
\hline CHH6 & 4 & $\mathrm{~F}$ & $218 \mathrm{~A}>\mathrm{G}$ & 17-bp dup at +3 & 48.5 & 2,950 & -3.1 & + & + & - \\
\hline CHH7 & 3 & M & $218 \mathrm{~A}>\mathrm{G}$ & 17-bp dup at +3 & 49.2 & 3,086 & -2.2 & + & + & - \\
\hline CHH8 & 3 & M & 17-bp dup at +3 & $182 \mathrm{G}>\mathrm{T}$ & 48.2 & 2,750 & -4.0 & + & - & + \\
\hline $\mathrm{NC} 1$ & 14 & $\mathrm{~F}$ & - & - & 47.2 & 2,650 & -4.8 & + & - & - \\
\hline $\mathrm{NC} 2$ & 3 & M & - & - & 40.0 & 2,840 & -7.0 & + & - & - \\
\hline NC3 & 8 & $\mathrm{~F}$ & - & - & ND & ND & -4.0 & + & - & + \\
\hline
\end{tabular}

$B H$ birth height (cm); $B W$ birth weight (g); $P H$ present height (SD); $S C$ skeletal change; $H H$ hair hypoplasia; $I D$ immunological disorder; $N D$ no data available

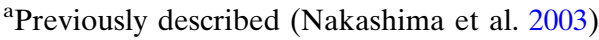

bde novo mutation

seen in just two patients, who were siblings. In most cases, birth length and height were not severely affected, although two patients who were short from birth developed very severe short stature $(<-8 \mathrm{SD})$. Patient $\mathrm{CHH} 4$, who had transcript mutations on both alleles, showed a mild phenotype.

Haplotype analysis of mutation alleles

Including the two previously screened cases $(\mathrm{CHH} 1$ and 2), the two recurrent mutations (17-bp dup at +3 and $218 \mathrm{G}>\mathrm{A}$ ) were found in five patients, respectively. Because these mutations are so common among Japanese $\mathrm{CHH}$ cases, we suspected the presence of founders. To investigate this possibility, we determined the haplotype structure of $R M R P$ in seven patients (CHH1, 2, 4-8) who had the common mutations.

Haplotypes were assessed by PCR direct sequencing, using the four SNPs within the RMRP region that showed minor allelic frequencies greater than $10 \%$ in the Japanese population (Nakashima et al. 2003; International HapMap Consortium 2005). Our analysis revealed that the two recurrent mutations were contained within distinct haplotypes (Fig. 1).

Next, we determined the haplotype block structure for the region surrounding $R M R P$ using genotype data from 44 Japanese individuals in the HapMap database. $R M R P$ was contained in a block spanning $\sim 20 \mathrm{~kb}$ (Fig. 2 top). The haplotype block was represented by seven haplotypes with $>1 \%$ frequency (Fig. 2 bottom). The haplotype of the chromosome containing the 17bp dup at +3 mutation was determined by parentalchildren transmission in three $(\mathrm{CHH} 1,5,8)$ of five disease chromosomes. All three had haplotype III
(AAAGG), which is seen in $13.6 \%$ of the Japanese population. The other two patients might have this haplotype, but the phase was not determined. Likewise, we determined the haplotype in two of five chromosomes containing the $218 \mathrm{~A}>\mathrm{G}$ mutation (CHH2 and $\mathrm{CHH} 5)$. Both had a rare haplotype (ATTGA) that was not seen among the 44 Japanese individuals used in the haplotype block analysis. The three other patients might also possess this haplotype, but the phase was not determined (Fig. 3).

\section{Discussion}

The mutation spectrum for $R M R P$ in the Japanese population is unique. The $70 \mathrm{~A}>\mathrm{G}$ founder mutation that is prevalent in Western populations (Ridanpaa et al. 2002) has not been reported in Japanese individuals. On the other hand, the $218 \mathrm{~A}>\mathrm{G}$ and 17 -bp dup at +3 mutations are common in Japanese patients, but have not been reported in other populations. We have shown that the two common mutations are contained within rare distinct haplotypes, indicating the presence of unique founders among Japanese $\mathrm{CHH}$ patients. Because these haplotypes have been defined by tag SNPs, our results will be useful in detecting $\mathrm{CHH}$ mutations and their carriers in Japanese $\mathrm{CHH}$ patients.

We saw no patient who possessed all of the skeletal, hair, and immunological features characteristic of classical $\mathrm{CHH}$. Extra-skeletal features are not prevalent in the Japanese population, although immunological disorders may yet develop in this young set of patients. As described previously (Nakashima et al. 2003), all patients were compound heterozygotes for 
Fig. 1 The haplotype structure of Japanese CHHs in the $R M R P$ region. The two common mutations are contained within distinct haplotypes (colored), respectively. SNP positions from the transcription start site of RMRP (denoted as +1 ) are indicated at the top. The telomere is to the left. Under the Chromosome heading, $F$ and $M$ indicate the paternal and maternal chromosomes, respectively

\begin{tabular}{|c|c|c|c|c|c|}
\hline & & & & $R M R P$ & \\
\hline Position (nt) & -149 & -58 & -48 & & 274 \\
\hline SNP & $\mathbf{T} / \mathbf{A}$ & $\mathrm{T} / \mathrm{C}$ & C/A & Mutation & $\mathbf{T} / \mathbf{C}$ \\
\hline Chromosome & & & & & \\
\hline CHH1/F & $\mathbf{T}$ & $\mathbf{T}$ & $\mathbf{C}$ & $17-b p$ dup at +3 & $\mathbf{T}$ \\
\hline CHH5/F & $\mathbf{T}$ & $\mathbf{T}$ & $\mathbf{C}$ & 17 -bp dup at +3 & $\mathbf{T}$ \\
\hline СНH6/M & $\mathbf{T}$ & $\mathbf{T}$ & $\mathbf{C}$ & 17 -bp dup at +3 & $\mathbf{T}$ \\
\hline CHH7/M & $\mathbf{T}$ & $\mathbf{T}$ & $\mathbf{C}$ & 17-bp dup at +3 & $\mathbf{T}$ \\
\hline CHH8/F & $\mathbf{T}$ & $\mathbf{T}$ & $\mathbf{C}$ & 17 -bp dup at +3 & $\mathbf{T}$ \\
\hline СHH2/M & $\mathbf{A}$ & C & $\mathbf{A}$ & $218 A>G$ & $\mathbf{T}$ \\
\hline CHH4/M & $\mathbf{A}$ & C & $\mathbf{A}$ & $218 A>G$ & $\mathbf{T}$ \\
\hline СНH5/M & $\mathbf{A}$ & C & $\mathbf{A}$ & $218 A>G$ & $\mathbf{T}$ \\
\hline CHH6/F & $\mathbf{A}$ & C & $\mathbf{A}$ & $218 A>G$ & $\mathbf{T}$ \\
\hline CHH7/F & $\mathbf{A}$ & C & $\mathbf{A}$ & $218 A>G$ & $\mathbf{T}$ \\
\hline СНH1M & $\mathbf{T}$ & $\mathbf{T}$ & $\mathbf{C}$ & $182 G>A$ & $\mathbf{T}$ \\
\hline CHH2/F & $\mathbf{A}$ & $\mathbf{C}$ & $\mathbf{A}$ & 17-bp ins at -20 & $\mathbf{T}$ \\
\hline CHH4/F & $\mathbf{T}$ & $\mathbf{T}$ & $\mathbf{A}$ & $217 \mathrm{C}>\mathrm{T}$ & $\mathbf{T}$ \\
\hline СHH8/M & $\mathbf{A}$ & $\mathbf{C}$ & $\mathbf{A}$ & $182 \mathrm{G}>\mathrm{T}$ & $\mathbf{T}$ \\
\hline
\end{tabular}

Fig. 2 The linkage disequilibrium $(L D)$ block and haplotype structure around $R M R P$ in the Japanese population. Top Genomic structure and the LD block containing RMRP. LD was evaluated using $\mathrm{D}^{\prime}$ statistical analysis. Bottom The haplotype structure and frequency of the LD block containing $R M R P$. The block was represented by seven haplotypes. *Tag SNPs: SNP1, rs10972549; SNP2, rs1538537; SNP3, 3750430; SNP8, rs4878625; SNP10, rs2071675

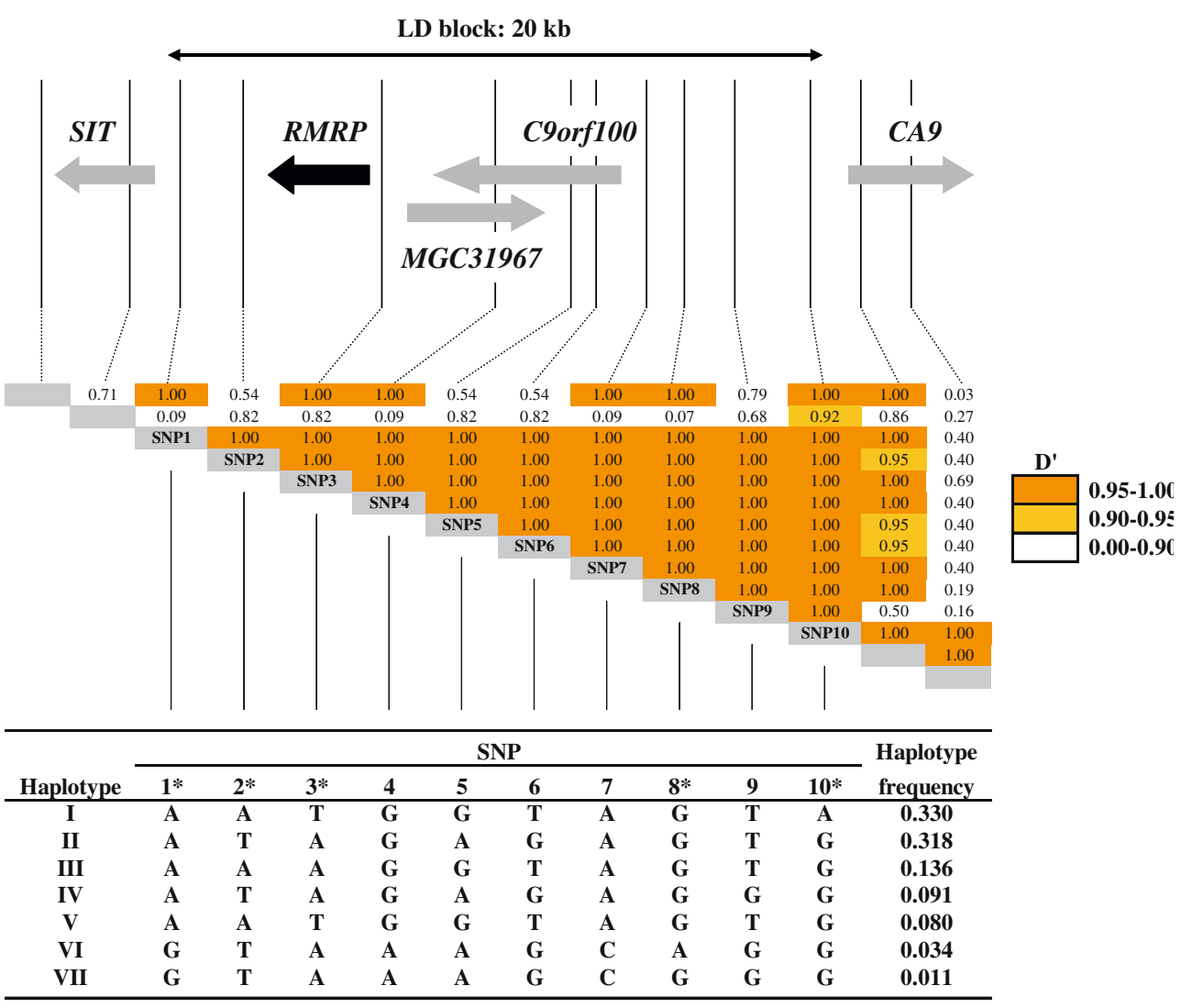

insertions or duplications in the promoter or $5^{\prime}$ transcribed regions and point mutations in the transcribed region. The former mutations cause marked decreases in gene transcription and, hence, the substantial effects on phenotype; in contrast, the latter mutations have only mild effects on transcription (Nakashima et al. 2003). This is consistent with the mild phenotype ob- served in patient $\mathrm{CHH} 4$, who had two point mutations. Although it is clear that $R M R P$ mutations produce a spectrum of phenotypes, from $\mathrm{CHH}$ variants with only skeletal manifestations (Bonafe et al. 2002) at the mild end to anauxetic dysplasia (Thiel et al. 2005) at the severe end, further studies are necessary to better delineate these phenotype-genotype correlations. 
Fig. 3 The haplotype structure of the region around $R M R P$. The two common mutations are contained within distinct haplotypes (colored). The telomere is to the right. SNP positions are indicated from the transcription start site of $R M R P$ (denoted as +1 )

\begin{tabular}{|c|c|c|c|c|c|c|}
\hline Position (nt) & 3536 & 845 & $R M R P$ & -149 & -11890 & -15877 \\
\hline Tag SNP & 1 & 2 & & 3 & 8 & 10 \\
\hline Chromosome & $\mathbf{A} / \mathbf{G}$ & $\mathbf{A} / \mathbf{T}$ & Mutation & $\mathbf{A} / \mathbf{T}$ & G/A & G/A \\
\hline CHH1/F & A & $\mathbf{A}$ & 17-bp dup at +3 & $\mathbf{A}$ & $\mathbf{G}$ & G \\
\hline CHH5/F & $\mathbf{A}$ & $\mathbf{A}$ & 17-bp dup at +3 & $\mathbf{A}$ & $\mathbf{G}$ & G \\
\hline CHH6/M & A & A & 17-bp dup at +3 & A & G & G/A \\
\hline CHH7/M & A & A & 17-bp dup at +3 & A & G & G/A \\
\hline CHH8/F & A & A & 17-bp dup at +3 & A & G & G \\
\hline CHH2/M & A & $\mathbf{T}$ & 218A $>G$ & $\mathbf{T}$ & G & A \\
\hline CHH4/M & $\mathbf{A}$ & $\mathbf{A} / \mathbf{T}$ & $218 A>G$ & $\mathbf{T}$ & G & G/A \\
\hline CHH5/M & A & $\mathbf{T}$ & $218 A>G$ & $\mathbf{T}$ & G & $\mathbf{A}$ \\
\hline CHH6/F & $\mathbf{A}$ & $\mathbf{T}$ & $218 A>G$ & $\mathbf{T}$ & G & G/A \\
\hline CHH7/F & A & $\mathbf{T}$ & $218 A>G$ & $\mathbf{T}$ & G & G/A \\
\hline CHH1M & A & A & $182 \mathrm{G}>\mathrm{A}$ & A & G & G \\
\hline CHH2/F & A & $\mathbf{T}$ & $17-b p$ ins at -20 & $\mathbf{T}$ & G & G \\
\hline CHH4/F & A & $\mathbf{A} / \mathbf{T}$ & $217 \mathrm{C}>\mathrm{T}$ & $\mathbf{A}$ & $\mathbf{G}$ & G/A \\
\hline CHH8/M & A & $\mathbf{T}$ & $182 G>T$ & $\mathbf{T}$ & G & A \\
\hline
\end{tabular}

Acknowledgments Eiji Nakashima, Hirofumi Ohashi, Gen Nishimura, and Shiro Ikegawa are members of the Japanese Skeletal Dysplasia Consortium, Tokyo, Japan. We thank the patients and their relatives who co-operated in this study. This work was supported by a grant-in-aid from Research on Child Health and Development (contract grant no. 17C-1).

\section{References}

Barrett JC, Fry B, Maller J, Daly MJ (2005) Haploview: analysis and visualization of LD and haplotype maps. Bioinformatics 21(2):263-265

Bonafe L, Schmitt K, Eich G, Giedion A, Superti-Furga A (2002) RMRP gene sequence analysis confirms a cartilagehair hypoplasia variant with only skeletal manifestations and reveals a high density of single-nucleotide polymorphisms. Clin Genet 61(2):146-151

Bonafe L, Dermitzakis ET, Unger S, Greenberg CR, CamposXavier BA, Zankl A, Ucla C, Antonarakis SE, SupertiFurga A, Reymond A (2005) Evolutionary comparison provides evidence for pathogenicity of $R M R P$ mutations. Plos Genet 1(4):e47

Castriota-Scanderbeg A, Dallapiccola B, Mingarelli R, Kozlowski K (2001) Distinctive metaphyseal chondrodysplasia simulating cartilage hair hypoplasia. Am J Med Genet 99(4):289-293

Harada D, Yamanaka Y, Ueda K, Shimizu J, Inoue M, Seino Y, Tanaka H (2005) An effective case of growth hormone treatment on cartilage-hair hypoplasia. Bone 36(2):317322

Hermanns P, Bertuch AA, Bertin TK, Dawson B, Schmitt ME, Shaw C, Zabel B, Lee B (2005) Consequences of mutations in the non-coding RMRP RNA in cartilage-hair hypoplasia. Hum Mol Genet 14(23):3723-3740

International HapMap Consortium (2005) A haplotype map of the human genome. Nature 437(7063):1299-1320
Kuijpers TW, Ridanpaa M, Peters M, de Boer I, Vossen JM, Kaitila I, Hennekam RC (2003) Short-limbed dwarfism with bowing, combined immune deficiency, and late onset aplastic anaemia caused by novel mutations in the RMPR gene. J Med Genet 40(10):761-766

Makitie O, Kaitila I (1993) Cartilage-hair hypoplasia-clinical manifestations in 108 Finnish patients. Eur J Pediatr 152(3):211-217

Nakashima E, Mabuchi A, Kashimada K, Onishi T, Zhang J, Ohashi H, Nishimura G, Ikegawa S (2003) RMRP mutations in Japanese patients with cartilage-hair hypoplasia. Am J Med Genet 123(3):253-256

Roifman CM, Gu Y, Cohen A (2006) Mutations in the RNA component of RNase mitochondrial RNA processing might cause Omenn syndrome. J Allergy Clin Immunol 117(4):897-903

Ridanpaa M, van Eenennaam H, Pelin K, Chadwick R, Johnson C, Yuan B, van Venrooij W, Pruijn G, Salmela R, Rockas S, Makitie O, Kaitila I, de la Chapelle A (2001) Mutations in the RNA component of RNase MRP cause a pleiotropic human disease, cartilage-hair hypoplasia. Cell 104(2):195-203

Ridanpaa M, Sistonen P, Rockas S, Rimoin DL, Makitie O, Kaitila I (2002) Worldwide mutation spectrum in cartilagehair hypoplasia: ancient founder origin of the major70$\mathrm{A} \rightarrow \mathrm{G}$ mutation of the untranslated $R M R P$. Eur $\mathrm{J}$ Hum Genet 10(7):439-447

Thiel CT, Horn D, Zabel B, Ekici AB, Salinas K, Gebhart E, Ruschendorf F, Sticht H, Spranger J, Muller D, Zweier C, Schmitt ME, Reis A, Rauch A (2005) Severely incapacitating mutations in patients with extreme short stature identify RNA-processing endoribonuclease $R M R P$ as an essential cell growth regulator. Am J Hum Genet 77(5):795806

Verloes A, Pierard GE, Le Merrer M, Maroteaux P (1990) Recessive metaphyseal dysplasia without hypotrichosis. A syndrome clinically distinct from McKusick cartilage-hair hypoplasia. J Med Genet 27(11):693-696 